\title{
The Scenario Approach to Robust Control Design
}

\author{
Giuseppe C. Calafiore and Marco C. Campi
}

\begin{abstract}
This paper proposes a new probabilistic solution framework for robust control analysis and synthesis problems that can be expressed in the form of minimization of a linear objective subject to convex constraints parameterized by uncertainty terms. This includes the wide class of NP-hard control problems representable by means of parameter-dependent linear matrix inequalities (LMIs). It is shown in this paper that by appropriate sampling of the constraints one obtains a standard convex optimization problem (the scenario problem) whose solution is approximately feasible for the original (usually infinite) set of constraints, i.e., the measure of the set of original constraints that are violated by the scenario solution rapidly decreases to zero as the number of samples is increased. We provide an explicit and efficient bound on the number of samples required to attain a-priori specified levels of probabilistic guarantee of robustness. A rich family of control problems which are in general hard to solve in a deterministically robust sense is therefore amenable to polynomial-time solution, if robustness is intended in the proposed risk-adjusted sense.
\end{abstract}

Index Terms-Probabilistic robustness, randomized algorithms, robust control, robust convex optimization, uncertainty.

\section{INTRODUCTION}

C ONVEX optimization, and semidefinite programming in particular, has become one of the mainstream frameworks for control analysis and synthesis. It is indeed well-known that standard linear control problems such as Lyapunov stability analysis and $H_{2}$ or $H_{\infty}$ synthesis may be formulated (and efficiently solved) in terms of solution of convex optimization problems with linear matrix inequality (LMI) constraints; see, for instance, [11], [25], and [42]. More recently, research in this field has concentrated on considering problems in which the data (for instance, the matrices describing a given plant) are uncertain. A "guaranteed" (or robust) approach in this case requires the satisfaction of the analysis or synthesis constraints for all admissible values (worst-case approach) of the uncertain parameters that appear in the problem data, see for instance [1]. Therefore, in the "robustified" version of the problem one has to determine a solution that satisfies a typically infinite number of convex constraints, generated by all the instances of the original constraints, as the uncertain parameters vary over their admissible domains.

Manuscript received March 18, 2004; revised December 9, 2004 and December 6, 2005. Recommended by Associate Editor E. Bai. This work was supported by FIRB funds of Italian Ministry of University and Research (MIUR) and by MIUR project New methods for Identification and Adaptive Control for Industrial Systems.

G. C. Calafiore is with the Dipartimento di Automatica e Informatica, Politecnico di Torino, 10129 Torino, Italy (e-mail: giuseppe.calafiore@polito.it).

M. C. Campi is with the Dipartimento di Elettronica per l'Automazione, Università di Brescia, 25123 Brescia, Italy (e-mail: marco.campi@ing.unibs.it).

Digital Object Identifier 10.1109/TAC.2006.875041
This "robust" convex programming paradigm has emerged around 1998 (see [7] and [28]) and, besides the systems and control areas, has found applications in, to mention but a few, truss topology design [6], robust antenna array design, portfolio optimization [29], and robust estimation [27]. Unfortunately, however, robust convex programs are not as easily solvable as standard ones, and are NP-hard in general, [7]. This implies for instance that - unlike standard semidefinite programs (SDP) - simply restating a control problem in the form of a robust SDP does not mean that the problem is amenable to efficient numerical solution.

The current state of the art for attacking robust convex optimization problems is by introducing suitable relaxations via "multipliers" or "scaling" variables, [9], [28], [41]. The main drawbacks of the relaxation approach are that the extent of the introduced conservatism is in general unknown (although it can be assessed for particular classes of problems, see [9], or asymptotically reduced by increasing the order of relaxation, [41]), and that the method itself can be applied only when the dependence of the data on the uncertainties has a particular and simple functional form, such as affine, polynomial or rational.

In this paper, we pursue a different probabilistic approach to robustness in control problems, in which the guarantees of performance are not intended in a deterministic sense (satisfaction against all possible uncertainty outcomes) but are instead intended in a probabilistic sense (satisfaction for most of the uncertainty instances, or in probability).

Introducing probability in robustness gained increasing interest in the literature in recent years, and the probabilistic approach is now a rather established methodology for robustness analysis; see, for instance, [3], [20], [33], [39], [43], and [44]. However, the probabilistic approach has found to date limited application in robust control synthesis. Basically, two different methodologies are currently available for probabilistic robust control synthesis: the approach based on the Vapnik-Chervonenkis theory of learning [34], [49], [50], and the sequential methods based on stochastic gradient iterations [19], [24], [37] or ellipsoid iterations, [31].

The first approach is very general but suffers from the conservatism of the Vapnik-Chervonenkis theory, [47], [48], which requires a very large number of randomly generated samples (i.e., it has high "sample complexity") in order to achieve the desired probabilistic guarantees. Even more importantly, the design methodology proposed in the seminal paper [49] does not aim to enforce the synthesis constraints in a robust sense, but is instead directed toward minimizing the average cost objective.

Alternatively, when the original synthesis problem is convex (which includes many, albeit not all, relevant control problems) the sequential approaches based on stochastic gradients [19], 
[24], [37] or ellipsoid iterations [31], may be applied with success. However, these methods are currently limited to feasibility problems, and have not yet been extended to deal satisfactorily with optimization. More fundamentally, these algorithms have asymptotic nature, i.e., they are guaranteed to converge to a robust feasible solution (if one exists) with probability one, but the total number of uncertainty samples that need to be drawn in order to achieve the desired solution cannot be fixed in advance.

The main contribution of the present work is to propose a general framework named "scenario approach" to solve convex robust control design problems. The fundamental idea is to consider only a finite number of sampled instances of the uncertainty affecting the system (the scenarios), and to solve in oneshot the corresponding standard convex problem. We will prove in this paper that the number of scenarios that need be considered is reasonably small and that the solution of the scenario problem has generalization properties, i.e., it satisfies with high probability also unseen scenarios. This is fundamentally different from the average reformulation proposed in [49].

In the key result of this paper (Theorem 1), we provide an efficient bound on the sample complexity of the scenario problem, that increases slowly with the required probabilistic levels. This is different from the stochastic sequential methods of [19], [24], [31], [37] that have an asymptotic nature. Moreover, a notable improvement upon the stochastic sequential methods is that our result holds for robust optimization problems and not only for feasibility.

This paper is organized as follows. In Section II, we introduce more precisely the notion of probabilistic design. Section III contains the main results on scenario optimization, while a proof of Theorem 1 is given in Section IV. Section V discusses some robust control problems that are amenable to the scenario-based solution, and presents a numerical example. Conclusions are finally drawn in Section VI.

\section{Worst-CASE Versus ProbabiListic Design}

A wide class of robust control problems can be expressed as optimization problems subject to constraints that are parameterized by the uncertainties affecting the plant. In formal terms, if $\theta \in \Theta \subseteq \mathbb{R}^{n_{\theta}}$ is the "design parameter" (which includes the actual controller parameters, plus possibly other additional variables such as parameters of Lyapunov functions, slack variables and scalings), and the family of admissible plants is parameterized by an "uncertainty vector" $\delta \in \Delta \subseteq \mathbb{R}^{n_{\delta}}$, then the prototype control problem we refer to consists in minimizing a linear objective $c^{T} \theta$ (the objective to be minimized can be taken as linear, without loss of generality), subject to that $\theta$ satisfies the constraints $f(\theta, \delta) \leq 0, \forall \delta \in \Delta$, where $f(\theta, \delta)$ : $\Theta \times \Delta \rightarrow[-\infty, \infty]$ is a scalar-valued function that specifies the constraints. Note that considering scalar-valued constraint functions is without loss of generality, since multiple constraints $f_{1}(\theta, \delta) \leq 0, \ldots, f_{n_{f}}(\theta, \delta) \leq 0$ can be reduced to a single scalar-valued constraint by the position $f(\theta, \delta) \doteq$ $\max _{i=1, \ldots, n_{f}} f_{i}(\theta, \delta)$. In typical situations, $\Delta$ has infinite cardinality, i.e., it contains an infinite number of possible instances for $\delta$.
In this paper, we make reference to problems where the $f$ function is convex in $\theta$, as formally stated in the next assumption.

Assumption 1 (Convexity): Let $\Theta \subseteq \mathbb{R}^{n_{\theta}}$ be a convex and closed set, and let $\Delta \subseteq \mathbb{R}^{n_{\delta}}$. We assume that $f(\theta, \delta): \Theta \times \Delta \rightarrow$ $[-\infty, \infty]$ is continuous and convex in $\theta$, for any fixed value of $\delta \in \Delta$.

Assumption 1 requires convexity only with respect to the design variable $\theta$, while generic nonlinear dependence with respect to $\delta$ is allowed.

Important special cases of robust convex programs are robust linear programs, [8], for which $f(\theta, \delta)$ is affine in $\theta$, and robust semidefinite programs, [9], [28], for which $f(\theta, \delta)=$ $\lambda_{\max }[F(\theta, \delta)]$, where

$$
F(\theta, \delta)=F_{0}(\delta)+\sum_{i=1}^{n_{\theta}} \theta_{i} F_{i}(\delta) \quad F_{i}(\delta)=F_{i}^{T}(\delta)
$$

and $\lambda_{\max }[\cdot]$ denotes the largest eigenvalue.

The reader is referred to Section $\mathrm{V}$ for a sample of robust control problems that fall within the convex framework here described.

a) Worst-Case Design: In worst-case design, one aims at enforcing the design constraint $f(\theta, \delta) \leq 0$ for all possible values of the uncertainty $\delta \in \Delta$. The resulting closed-loop system will hence exhibit a performance level that is guaranteed for each and every plant in the uncertain family. However, a fundamental problem is encountered along this approach: Obtaining worst-case solutions has been proven to be computationally hard; explicit results on the NP-hardness of several worst-case design problems are for instance found in [10], [13], and [36]. In addition, a second criticism applies to a worst-case design: Seeking guarantees against the worst-case can introduce undesirable conservatism in the design, since all the design focus is on a special "ill" situation (the worst-case plant), which could as well be unrepresentative of the majority of admissible plants. These critical points that we recalled here are extensively documented in [49], to which we refer the reader. For these reasons, we adopt in this paper an alternative design approach, based on satisfaction of the design constraints in probability.

b) Probabilistic Robust Design: In the probabilistic design paradigm, we assume a probability measure Prob over the uncertainty set $\Delta$ is given. Then, for a given probability level $\epsilon \in(0,1)$, we seek a design parameter $\theta$ that minimizes $c^{T} \theta$ while satisfying all constraints but a small fraction of them whose probability is no larger than the prespecified level $\epsilon$. It should be noted that this approach can be seen as a relaxation of the worst-case paradigm where one allows a risk level $\epsilon$ and looks for a design parameter such that the performance specification is violated by at most a fraction $\epsilon$ of the plants in the uncertainty family.

\section{SCENARIO OPTIMIZATION}

The main result of this section (Theorem 1) shows that a solution to the probabilistic design problem can be found at low computational effort, with complete generality. 
We start by formally introducing some relevant definitions. We assume that the support $\Delta$ for $\delta$ is endowed with a $\sigma$-algebra $\mathcal{D}$ and that Prob is defined over $\mathcal{D}$. Moreover, we assume that $\{\delta \in \Delta: f(\theta, \delta) \leq 0\} \in \mathcal{D}, \forall \theta \in \Theta$. Depending on the situation at hand, Prob can have different interpretations. Sometimes, it is the actual probability with which the uncertainty parameter $\delta$ takes on value in $\Delta$. Other times, Prob simply describes the relative importance we attribute to different uncertainty instances. We have the following definition.

Definition 1 (Probability of Violation): Let $\theta \in \Theta$ be given. The probability of violation of $\theta$ is defined as

$$
V(\theta) \doteq \operatorname{Prob}\{\delta \in \Delta: f(\theta, \delta)>0\}
$$

For example, if a uniform (with respect to Lebesgue measure) probability density is assumed, then $V(\theta)$ measures the volume of "bad" parameters $\delta$ such that the constraint $f(\theta, \delta) \leq 0$ is violated. Clearly, a solution $\theta$ with small associated $V(\theta)$ is feasible for most of the problem instances, i.e., it is approximately feasible for the robust problem. This concept of approximate feasibility has been introduced in the context of robust control in [4]. Any solution $\theta$ such that $V(\theta) \leq \epsilon$ is here named an " $\epsilon$-level" solution.

Definition 2 ( $\epsilon$-Level Solution): Let $\epsilon \in(0,1)$. We say that $\theta \in \Theta$ is an $\epsilon$-level robustly feasible (or, more simply, an $\epsilon$-level) solution, if $V(\theta) \leq \epsilon$.

Our goal is to devise an algorithm that returns a $\epsilon$-level solution, where $\epsilon$ is any fixed small level, and that is worst-case optimal over the set of satisfied constraints. To this purpose, we now introduce the "scenario" version of the robust design problem. By scenario it is here meant any realization or instance of the uncertainty parameter $\delta$. In the scenario design we optimize the objective subject to a finite number of randomly selected scenarios. Later on in Theorem 1 we show that the scenario design permits to solve the $\epsilon$-level probabilistic design.

Definition 3 (Scenario Design): Assume that $N$ independent identically distributed samples $\delta^{(1)}, \ldots, \delta^{(N)}$ are drawn according to probability Prob. A scenario design problem is given by the convex program

$\operatorname{RCP}_{N}: \min _{\theta \in \Theta} c^{T} \theta \quad$ subject to $: f\left(\theta, \delta^{(i)}\right) \leq 0, i=1, \ldots, N$.

The acronym $\operatorname{RCP}_{N}$ refers to the fact that (1) is a robust convex program with $N$ constraints.

To avoid mathematical clutter, we here make the following technical assumption on the scenario problem. However, this assumption can be released, as shown in Appendix A.

Assumption 2: For all possible extractions $\delta^{(1)}, \ldots, \delta^{(N)}$, the optimization problem (1) is either unfeasible, or, if feasible, it attains a unique optimal solution.

Contrary to the robust convex problem, the scenario problem $\mathrm{RCP}_{N}$ is a standard convex optimization problem with a finite number $N$ of constraints and, hence, its optimal solution $\hat{\theta}_{N}$ is usually efficiently computable by means of numerical algorithms. Moreover, since only $N$ constraints are imposed in
$\mathrm{RCP}_{N}$, it is clear that the optimal solution of $\mathrm{RCP}_{N}$ is superoptimal for the robust convex problem with all constraints in place, i.e., the objective corresponding to $\hat{\theta}_{N}$ outperforms the one achieved with the solution of the robust convex program. In this way, the scenario approach alleviates the conservatism of the worst-case approach.

The fundamental question that need now be addressed is: what guarantee can be provided on the level of feasibility of the solution $\hat{\theta}_{N}$ of $\operatorname{RCP}_{N}$ ? The following key Theorem 1 and its Corollary 1 answer this question.

Before stating the theorem, we note that, since the constraints $f\left(\theta, \delta^{(i)}\right) \leq 0$ are randomly selected, the resulting optimal solution $\hat{\theta}_{N}$ is a random variable that depends on the multi-sample extraction $\left(\delta^{(1)}, \ldots, \delta^{(N)}\right)$. Therefore, $\hat{\theta}_{N}$ can be a $\epsilon$-level solution for a given random extraction and not for another. In the theorem, the parameter $\beta$ bounds the probability that $\hat{\theta}_{N}$ is not a $\epsilon$-level solution. Thus, $\beta$ is the risk of failure, or confidence, associated to the randomized solution algorithm.

Theorem 1: Let Assumption 2 be satisfied. Fix two real numbers $\epsilon \in(0,1)$ (level parameter) and $\beta \in(0,1)$ (confidence parameter). If

$$
\begin{aligned}
N & \geq N_{\text {gen }}(\epsilon, \beta) \\
& \left.\doteq \inf _{\nu \in(0,1)} \frac{1}{1-\nu}\left(\frac{1}{\epsilon} \ln \frac{1}{\beta}+n_{\theta}+\frac{n_{\theta}}{\epsilon} \ln \frac{1}{\nu \epsilon}+\frac{1}{\epsilon} \ln \frac{\left(\frac{n_{\theta}}{\mathrm{e}}\right)^{n_{\theta}}}{n_{\theta} !}\right)\right]
\end{aligned}
$$

( $\lceil\cdot\rceil$ denotes the smallest integer greater than or equal to the argument) then, with probability no smaller than $1-\beta$, either the scenario problem $\mathrm{RCP}_{N}$ is unfeasible and, hence, also the initial robust convex program is unfeasible; or, $\mathrm{RCP}_{N}$ is feasible, and then its optimal solution $\hat{\theta}_{N}$ is $\epsilon$-level robustly feasible. *

In this theorem, probability $1-\beta$ refers to the $N$-fold probability $\operatorname{Prob}^{N}(=$ Prob $\times \cdots \times$ Prob, $N$ times $)$ in $\Delta^{N}=$ $\Delta \times \Delta \times \cdots \times \Delta$, which is the set to which the extracted multisample $\left(\delta^{(1)}, \ldots, \delta^{(N)}\right)$ belongs. Here and elsewhere, the measurability of $\left\{V\left(\hat{\theta}_{N}\right) \leq \epsilon\right\}$, as well as that of other sets in $\Delta^{N}$, is taken as an assumption. A proof of Theorem 1 is given in Section IV. A visual interpretation of this theorem is provided in Fig. 1.

Theorem 1 states that if $N$ (specified by (2)) random scenarios are drawn, the optimal solution of $\mathrm{RCP}_{N}$ is $\epsilon$-level feasible according to Definition 2 , with high probability $1-\beta$. Parameter $\beta$ is important in theory since, if $\beta$ is pushed down to zero, $N$ goes to infinity. However, for a practical use, we can observe that $\beta$ plays a very marginal role. The reason is that $\beta$ shows up in (2) under the sign of logarithm so that it can be made very small $\left(10^{-10}\right.$ or even $\left.10^{-20}\right)$ without significantly increasing $N$. The scenario approach thus provides us with a viable and implementable way to robustify a nominal design up to a desired level $\epsilon$.

Bound (2) can be simplified as stated in the next corollary.

Corollary 1: The results in Theorem 1 hold for

$$
N \geq N_{\log }(\epsilon, \beta) \doteq\left\lceil\frac{2}{\epsilon} \ln \frac{1}{\beta}+2 n_{\theta}+\frac{2 n_{\theta}}{\epsilon} \ln \frac{2}{\epsilon}\right\rceil .
$$




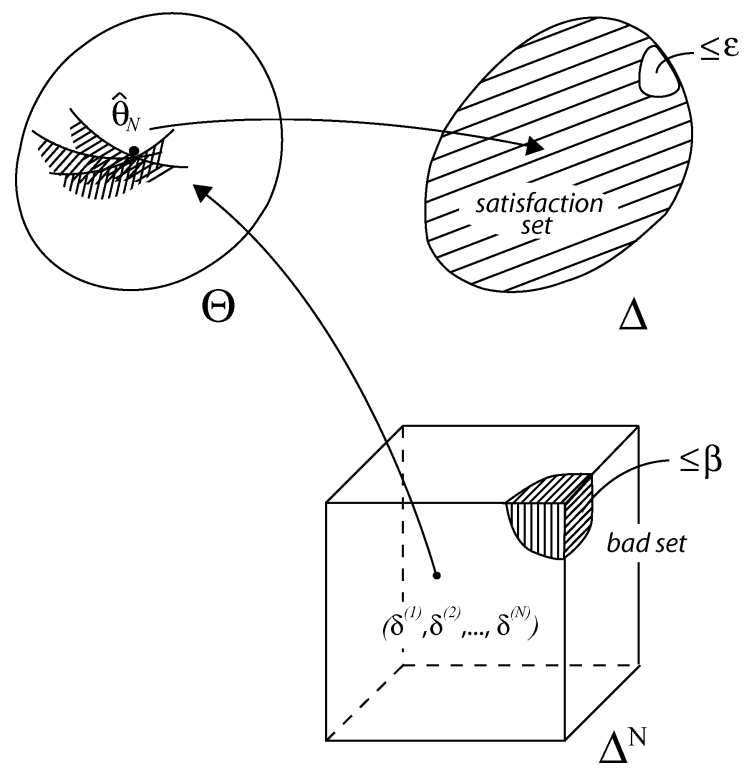

Fig. 1. Interpretation of Theorem 1: with probability at least $1-\beta$ we extract a multisample $\delta^{(1)}, \ldots, \delta^{(N)}$ from outside the "bad set." The resulting optimal solution $\hat{\theta}_{N}$ is, hence, feasible for all but at most a set of measure $\epsilon$ of the uncertainties.

The subscript "log" in (3) highlights the logarithmic dependence on $1 / \beta$.

Proof: Observe that $\left(n_{\theta} / \mathrm{e}\right)^{n_{\theta}} \leq n_{\theta}$ ! and, hence, the last term in (2) is nonpositive and can be dropped, leading to

$$
N_{\text {gen }}(\epsilon, \beta) \leq\left\lceil\frac{1}{1-\nu}\left(\frac{1}{\epsilon} \ln \frac{1}{\beta}+n_{\theta}+\frac{n_{\theta}}{\epsilon} \ln \frac{1}{\nu \epsilon}\right)\right\rceil
$$

where $\nu$ can be freely selected in $(0,1)$. The statement of the corollary is then obtained by selecting $\nu=1 / 2$ in (4). We also note that further optimizing (4) with respect to $\nu$ always leads to a $\nu \leq 1 / 2$, with a corresponding improvement by at most of a factor 2 .

Some remarks further comment the results in Theorem 1 and Corollary 1.

Remark 1 (The Role of Convexity): Theorem 1 says that if we extract a finite number $N$ of constraints, then the solution of the randomized problem-if feasible-satisfies most of the other unseen constraints. This is a generalization property in the learning theoretic sense: The explicit satisfaction of some "training" scenarios generalizes automatically to the satisfaction of other unseen scenarios. It is interesting to note that generalization calls for some kind of structure, and the only structure used here is convexity. So, convexity in the scenario approach is fundamental in two different respects: On the computational side, it allows for an efficient solution of the ensuing optimization problem; on the theoretical side, it allows for generalization.

Remark 2 (Sample Complexity): Formula (3) provides a ready-to-use "sample complexity" for the number $N$ of random scenarios that need to be drawn in order to achieve the desired probabilistic level in the solution. In fact $N_{\log }(\epsilon, \beta)$ in (3) only depends on the number $n_{\theta}$ of optimization variables, besides the probabilistic levels $\epsilon$ and $\beta$, and its evaluation does not involve computing complicated complexity measures such as the
VC-dimension. It is not difficult to conceive situations where the class of sets $\{\delta \in \Delta: f(\theta, \delta)>0\} \subseteq \Delta$, parameterized in $\theta$, has infinite VC-dimension (see, e.g., [47] for a definition of VC-dimension), even for small $n_{\theta}$. Then, estimating $\operatorname{Prob}\{\delta \in \Delta: f(\theta, \delta)>0\}=V(\theta)$ uniformly with respect to $\theta$ is impossible and the VC-theory is of no use. Theorem 1 says that, if attention is restricted to $\hat{\theta}_{N}$, then estimating $V\left(\hat{\theta}_{N}\right)$ is indeed possible and this can be done at a low computational cost.

Remark 3 (Prob-Independent Bound): In some applications, probability Prob is not explicitly known, and the scenarios are directly made available as "observations." This could for example be the case when the instances of $\delta$ are actually related to various measurements or identification experiments made on a plant at different times and/or different operating conditions; see, e.g., [15] and [16]. In this connection, we notice that the bound (2) is probability independent, i.e., it holds irrespective of the underlying probability Prob, and can therefore be applied even when Prob is unknown.

Remark 4 (Comparison With an Older Bound): In a previous work [17] devoted to general convex optimization, the authors of the present paper derived a different bound on the sample complexity of sampled convex programs. This earlier result establishes that the statement of Theorem 1 holds for

$$
N \geq N_{\operatorname{lin}}(\epsilon, \beta) \doteq\left\lceil\frac{n_{\theta}}{\epsilon \beta}-1\right\rceil .
$$

This bound is linear both in $\epsilon^{-1}$ and $\beta^{-1}$ and, since one typically desires to have $\beta$ very small, bound (5) is worse by orders of magnitude with respect to the newly derived bounds. The number of samples required by (2) appears to be reasonable and useful for a practical implementation of our method. For instance, in a problem with $n_{\theta}=10$ variables, setting probability levels $\epsilon=0.01$ and $\beta=10^{-5}$, bound (2) would require $N_{\text {gen }}=8725$ samples.

\section{A. The Chance-Constrained Problem}

Consider the probability-constrained problem

$$
\begin{array}{ll}
\operatorname{CCP}(\epsilon): \min _{\theta \in \Theta} c^{T} \theta & \text { subject to : } \\
& \operatorname{Prob}\{\delta \in \Delta: f(\theta, \delta) \leq 0\} \geq 1-\epsilon .
\end{array}
$$

The distinctive feature of $\operatorname{CCP}(\epsilon)$ is that it is required that the neglected constraint set is chosen in an optimal way, i.e., among all sets of constraints with probability no larger than $\epsilon$, the removed one is the one that allows for the greatest reduction in the design objective. In the optimization literature, this problem is called a "chance-constrained" optimization problem, see e.g., [38], [46]. It should readily be remarked that an exact numerical solution of $\operatorname{CCP}(\epsilon)$ is in general hopeless, see [38], [46]. Moreover, $\mathrm{CCP}(\epsilon)$ is in general nonconvex, even when the function $f(\theta, \delta)$ is convex in $\theta$ for all $\delta \in \Delta$. There are however very specialized instances of chance-constrained problems (in particular, linear programs with log-concave distributions of the uncertain parameters) that can indeed be reformulated as standard convex programs and, hence, solved efficiently, see again [38], and [35] for an application to control design. 
As we have already seen, $\mathrm{RCP}_{N}$ returns with high probability a feasible solution of $\operatorname{CCP}(\epsilon)$. In the next theorem, we establish a precise connection between $\operatorname{CCP}(\epsilon)$ and $\operatorname{RCP}_{N}$.

Theorem 2: Let $\epsilon, \beta \in(0,1)$ be given probability levels. Let $J_{\mathrm{CCP}(\epsilon)}$ denote the optimal objective value of the chanceconstrained problem $\operatorname{CCP}(\epsilon)$ in (6) when it is feasible (i.e., $J_{\mathrm{CCP}(\epsilon)} \doteq \inf _{\theta \in \Theta} c^{T} \theta$ subject to $\left.V(\theta) \leq \epsilon\right)$ and let $J_{\mathrm{RCP}_{N}}$ be the optimal objective value of the scenario problem $\operatorname{RCP}_{N}$ in (1) when it is feasible (notice that $J_{\mathrm{RCP}_{N}}$ is a random variable, while $J_{\mathrm{CCP}(\epsilon)}$ is a deterministic value), with $N$ any number satisfying (2). Then

1) with probability at least $1-\beta$, if $\operatorname{RCP}_{N}$ is feasible it holds that

$$
J_{\mathrm{RCP}_{N}} \geq J_{\mathrm{CCP}(\epsilon)}
$$

2) assume $\operatorname{CCP}\left(\epsilon_{1}\right)$ is feasible, where $\epsilon_{1}=1-(1-$ $\beta)^{1 / N}$. With probability at least $1-\beta$, it holds that

$$
J_{\mathrm{RCP}_{N}} \leq J_{\mathrm{CCP}\left(\epsilon_{1}\right)}
$$

A proof of this theorem is given in Appendix B.

A few words help clarify result 2 in Theorem 2 . First notice that $J_{\mathrm{CCP}(\epsilon)}$ is a nonincreasing function of $\epsilon$. Result 2 states that the optimal value $J_{\mathrm{RCP}_{N}}$ (where $N$ has been selected so that the optimal solution is $\epsilon$-level feasible with probability $1-\beta$ ) is, with probability at least $1-\beta$, no worse than $J_{\mathrm{CCP}\left(\epsilon_{1}\right)}$, for a certain $\epsilon_{1} \leq \epsilon$ explicitly given. For a ready comparison between $\epsilon$ and $\epsilon_{1}$, observe that relation $a^{s} \leq s a+(1-s)$ holds for any $a \geq 0$ and $0 \leq s \leq 1$ (as it easily follows by observing that the two sides coincide for $s=0$ and $s=1$ and that $a^{s}$ is convex in $s)$. Then, with the position $a \doteq 1-\beta ; s \doteq 1 / N$, we have

$$
\epsilon_{1}=1-(1-\beta)^{1 / N} \geq 1-\left[\frac{1}{N}(1-\beta)+\left(1-\frac{1}{N}\right)\right]=\frac{\beta}{N}
$$

which, used in result 2 of the theorem, gives $J_{\mathrm{RCP}_{N}} \leq$ $J_{\mathrm{CCP}(\beta / N)}$, with $N$ any number satisfying (2). For a crude evaluation, use bound (3) for $N$. If $n_{\theta}>1$ and $\beta$ is not taken to be very small as compared to $\epsilon$, then the dominant term in (3) is $\left(2 n_{\theta} / \epsilon\right) \ln (2 / \epsilon)$, leading to $\epsilon_{1} \approx \beta / N \approx\left(\beta / 2 n_{\theta} \ln (2 / \epsilon)\right) \epsilon$, where $\beta / 2 n_{\theta} \ln (2 / \epsilon)$ is the rescaling factor between $\epsilon$ and $\epsilon_{1}$.

\section{PRELIMINARIES AND PROOF OF THEOREM 1}

\section{A. Preliminaries}

We first recall a classical result due to Helly; see [40].

Lemma 1 (Helly): Let $\left\{\mathcal{X}_{i}\right\}_{i=1, \ldots, p}$ be a finite collection of convex sets in $\mathbb{R}^{n}$. If every sub-collection consisting of $n+1$ sets has a nonempty intersection, then the entire collection has a nonempty intersection.

Next, we prove a key instrumental result. Consider the convex optimization program

$$
\mathcal{P}: \min _{x \in \mathbb{R}^{n}} c^{T} x \quad \text { subject to }: x \in \bigcap_{i \in\{1, \ldots, m\}} \mathcal{X}_{i}
$$

where $\mathcal{X}_{i}, i=1, \ldots, m$, are closed convex sets, and define the convex programs $\mathcal{P}_{k}, k=1, \ldots, m$, obtained from $\mathcal{P}$ by removing the $k$ th constraint

$$
\mathcal{P}_{k}: \min _{x \in \mathbb{R}^{n}} c^{T} x \quad \text { subject to }: x \in \bigcap_{i \in\{1, \ldots, m\} \backslash k} \mathcal{X}_{i}
$$

Assume program $\mathcal{P}$ and the programs $\mathcal{P}_{k}$ admit a unique optimal solution, say $x^{*}$ and $x_{k}^{*}$, respectively, and let $J^{*}=c^{T} x^{*}$ and $J_{k}^{*}=c^{T} x_{k}^{*}$. We have the following definition.

Definition 4 (Support Constraint): The $k$ th constraint $\mathcal{X}_{k}$ is a support constraint for $\mathcal{P}$ if $J_{k}^{*}<J^{*}$.

The following theorem holds.

Theorem 3: The number of support constraints for problem $\mathcal{P}$ is at most $n$.

A proof of this result was first given by the authors of the present paper in [17]. We here report an alternative and more compact proof based on an idea suggested to us by A. Nemirovski in a personal communication.

Proof: Let problem $\mathcal{P}$ have $q$ support constraints $\mathcal{X}_{s_{1}}, \ldots, \mathcal{X}_{s_{q}}$, where $\mathcal{S} \doteq\left\{s_{1}, \ldots, s_{q}\right\}$ is a subset of $q$ indexes from $\{1, \ldots, m\}$. We next prove (by contradiction) that $q \leq n$.

Consider the smallest objective improvement obtained by removing a support constraint $\eta_{\min } \doteq \min _{k \in \mathcal{S}}\left(J^{*}-J_{k}^{*}\right)$ and, for some $\eta$ with $0<\eta<\eta_{\min }$, define the hyperplane $\mathcal{H} \doteq\{x$ : $\left.c^{T} x=J^{*}-\eta\right\}$. By construction, the $q$ points $x_{k}^{*}, k \in \mathcal{S}$, lie in the half-space $\left\{x: c^{T} x<J^{*}-\eta\right\}$, while $x^{*}$ lies in the half-space $\left\{x: c^{T} x>J^{*}-\eta\right\}$, and therefore $\mathcal{H}$ separates $x_{k}^{*}$, $k \in \mathcal{S}$, from $x^{*}$. Next, for all indices $k \in \mathcal{S}$, we denote with $\bar{x}_{k}^{*}$ the point of intersection between the line segment $\overline{x_{k}^{*} x^{*}}$ and $\mathcal{H}$.

Since $x_{k}^{*} \in \bigcap_{i \in\{1, \ldots, m\} \backslash k} \mathcal{X}_{i}, k \in \mathcal{S}$, and $x^{*} \in$ $\bigcap_{i \in\{1, \ldots, m\}} \mathcal{X}_{i}$, then by convexity we have that $\bar{x}_{k}^{*} \in$ $\bigcap_{i \in\{1, \ldots, m\} \backslash k} \mathcal{X}_{i}, k \in \mathcal{S}$ and, therefore, (since, by construction, $\bar{x}_{k}^{*} \in \mathcal{H}$ )

$$
\bar{x}_{k}^{*} \in\left(\bigcap_{i \in\{1, \ldots, m\} \backslash k} \mathcal{X}_{i}\right) \bigcap \mathcal{H}, \quad k \in \mathcal{S} .
$$

For $i=1, \ldots, m$, define the convex sets $\Omega_{i} \doteq \mathcal{X}_{i} \bigcap \mathcal{H}$, and consider any collection $\left\{\Omega_{i_{1}}, \ldots, \Omega_{i_{n}}\right\}$ of $n$ of these sets.

Suppose now (for the purpose of contradiction) that $q>n$. Then, there must exist an index $j \notin\left\{i_{1}, \ldots, i_{n}\right\}$ such that $\mathcal{X}_{j}$ is a support constraint and, by the previous reasoning, this means that there exists a point $\bar{x}_{j}^{*}$ such that $\bar{x}_{j}^{*} \in\left(\bigcap_{i \in\{1, \ldots, m\} \backslash j} \mathcal{X}_{i}\right) \cap \mathcal{H}$. Thus, $\bar{x}_{j}^{*} \in \Omega_{i_{1}} \cap \cdots \cap \Omega_{i_{n}}$, that is the collection of convex sets $\left\{\Omega_{i_{1}}, \ldots, \Omega_{i_{n}}\right\}$ has at least a point in common. Now, since the sets $\Omega_{i}, i=1, \ldots, m$, belong to the hyperplane $\mathcal{H}$ (i.e., to $\mathbb{R}^{n-1}$, modulo a fixed translation) and all collections composed of $n$ of these sets have a point in common, by Helly's Lemma (Lemma 1) there exists a point $\tilde{x}$ such that $\tilde{x} \in \bigcap_{i \in\{1, \ldots, m\}} \Omega_{i}$. Such a $\tilde{x}$ would, therefore, be feasible for problem $\mathcal{P}$; moreover, it would yield an objective value $\tilde{J}=c^{T} \tilde{x}<c^{T} x^{*}=J^{*}$ (since $\tilde{x} \in \mathcal{H}$ ). This is a contradiction, because $x^{*}$ would no longer be an optimal solution for $\mathcal{P}$, and, hence, we conclude that $q \leq n$.

We are now ready to present a proof of Theorem 1 . 


\section{B. Proof of Theorem 1}

The fact that, if $\mathrm{RCP}_{N}$ is unfeasible, then the initial robust convex program is unfeasible too is trivially true, since the latter program exhibits more constraints than $\mathrm{RCP}_{N}$. Thus, we have to prove that, with probability $1-\beta$, either $\operatorname{RCP}_{N}$ is unfeasible or, if feasible, its solution is $\epsilon$-level robustly feasible. This part of the proof is inspired by a similar proof given in a different context in [23].

For clarity of exposition, we first assume that problem $\mathrm{RCP}_{N}$ is feasible for any selection of $\delta^{(1)}, \ldots, \delta^{(N)}$. The case where infeasibility can occur is obtained as an easy extension as indicated at the end of the proof.

Given $N$ scenarios $\delta^{(1)}, \ldots, \delta^{(N)}$, select a subset $I=\left\{i_{1}, \ldots, i_{n_{\theta}}\right\}$ of $n_{\theta}$ indices from $\{1, \ldots, N\}$ and let $\hat{\theta}_{I}$ be the optimal solution of the program

$$
\min _{\theta \in \Theta} c^{T} \theta \quad \text { subject to : } f\left(\theta, \delta^{\left(i_{j}\right)}\right) \leq 0, j=1, \ldots, n_{\theta} \text {. }
$$

Based on $\hat{\theta}_{I}$ we next introduce a subset $\Delta_{I}^{N}$ of the set $\Delta^{N}$ defined as

$$
\Delta_{I}^{N} \doteq\left\{\left(\delta^{(1)}, \ldots, \delta^{(N)}\right): \hat{\theta}_{I}=\hat{\theta}_{N}\right\}
$$

$\left(\hat{\theta}_{N}\right.$ is the optimal solution with all $N$ constraints $\delta^{(1)}, \ldots, \delta^{(N)}$ in place).

Let now $I$ range over the collection $\mathcal{I}$ of all possible choices of $n_{\theta}$ indexes from $\{1, \ldots, N\}$ ( $\mathcal{I}$ contains $\left(\begin{array}{c}N \\ n_{\theta}\end{array}\right)$ sets $)$. We want to prove that

$$
\Delta^{N}=\bigcup_{I \in \mathcal{I}} \Delta_{I}^{N}
$$

To show (8), take any $\left(\delta^{(1)}, \ldots, \delta^{(N)}\right) \in \Delta^{N}$. From the set of constraint $\delta^{(1)}, \ldots, \delta^{(N)}$ eliminate a constraint which is not a support constraint (this is possible in view of Theorem 3 , since $\left.N>n_{\theta}\right)$. The resulting optimization problem with $N-1$ constraints admits the same optimal solution $\hat{\theta}_{N}$ as the original problem with $N$ constraints. Consider now the set of the remaining $N-1$ constraints and, among these, remove a constraint which is not a support constraint for the problem with $N-1$ constraints. Again, the optimal solution does not change. If we keep going this way until we are left with $n_{\theta}$ constraints, in the end we still have $\hat{\theta}_{N}$ as optimal solution, showing that $\left(\delta^{(1)}, \ldots, \delta^{(N)}\right) \in \Delta_{I}^{N}$, where $I$ is the set containing the $n_{\theta}$ constraints remaining at the end of the process. Since this is true for any choice of $\left(\delta^{(1)}, \ldots, \delta^{(N)}\right) \in \Delta^{N},(8)$ is proven. Next, let $B \doteq\left\{\left(\delta^{(1)}, \ldots, \delta^{(N)}\right): V\left(\hat{\theta}_{N}\right)>\epsilon\right\}$ and $B_{I} \doteq$ $\left\{\left(\delta^{(1)}, \ldots, \delta^{(N)}\right): V\left(\hat{\theta}_{I}\right)>\epsilon\right\}$. We now have

$$
\begin{aligned}
B & =B \cap \Delta^{N} \\
& =B \cap\left(\cup_{I \in \mathcal{I}} \Delta_{I}^{N}\right) \quad(\text { apply }(8)) \\
& =\cup_{I \in \mathcal{I}}\left(B \cap \Delta_{I}^{N}\right) \\
& \left.=\cup_{I \in \mathcal{I}}\left(B_{I} \cap \Delta_{I}^{N}\right) . \quad \text { (because of }(7)\right) .
\end{aligned}
$$

A bound for $\operatorname{Prob}^{N}(B)$ is now obtained by bounding $\operatorname{Prob}\left(B_{I} \cap \Delta_{I}^{N}\right)$ and then summing over $I \in \mathcal{I}$.

Fix any $I$, e.g., $I=\left\{1, \ldots, n_{\theta}\right\}$ to be more explicit. The set $B_{I}=B_{\left\{1, \ldots, n_{\theta}\right\}}$ is in fact a cylinder with base in the cartesian product of the first $n_{\theta}$ constraint domains (this follows from the fact that condition $V\left(\hat{\theta}_{\left\{1, \ldots, n_{\theta}\right\}}\right)>\epsilon$ only involves the first $n_{\theta}$ constraints). Fix $\left(\bar{\delta}^{(1)}, \ldots, \bar{\delta}^{\left(n_{\theta}\right)}\right) \in$ base of the cylinder. For a point $\left(\bar{\delta}^{(1)}, \ldots, \bar{\delta}^{\left(n_{\theta}\right)}, \delta^{\left(n_{\theta}+1\right)}, \ldots, \delta^{(N)}\right)$ to be in $B_{\left\{1, \ldots, n_{\theta}\right\}} \cap$ $\Delta_{\left\{1, \ldots, n_{\theta}\right\}}^{N}$, constraints $\delta^{\left(n_{\theta}+1\right)}, \ldots, \delta^{(N)}$ must be satisfied by $\hat{\theta}_{\left\{1, \ldots, n_{\theta}\right\}}$, for, otherwise, we would not have $\hat{\theta}_{\left\{1, \ldots, n_{\theta}\right\}}=\hat{\theta}_{N}$, as it is required in $\Delta_{\left\{1, \ldots, n_{\theta}\right\}}^{N}$. However, $V\left(\hat{\theta}_{\left\{1, \ldots, n_{\theta}\right\}}\right)>\epsilon$ in $B_{\left\{1, \ldots, n_{\theta}\right\}}$. Thus, by the fact that the extractions are independent, we conclude that the equation shown at the bottom of the page holds. The probability on the left-hand side is nothing but the conditional probability that $\left(\delta^{(1)}, \ldots, \delta^{(N)}\right) \in B_{\left\{1, \ldots, n_{\theta}\right\}} \cap$ $\Delta_{\left\{1, \ldots, n_{\theta}\right\}}^{N}$ given $\delta^{(1)}=\bar{\delta}^{(1)}, \ldots, \delta^{\left(n_{\theta}\right)}=\bar{\delta}^{\left(n_{\theta}\right)}$. Integrating over the base of the cylinder $B_{\left\{1, \ldots, n_{\theta}\right\}}$, we then obtain

$$
\begin{aligned}
\operatorname{Prob}^{N}\left(B_{\left\{1, \ldots, n_{\theta}\right\}} \cap \Delta_{\left\{1, \ldots, n_{\theta}\right\}}^{N}\right) \\
<(1-\epsilon)^{N-n_{\theta}} \cdot \operatorname{Prob}^{n_{\theta}}\left(\text { base of } B_{\left\{1, \ldots, n_{\theta}\right\}}\right) \\
\leq(1-\epsilon)^{N-n_{\theta}} .
\end{aligned}
$$

From (9), we finally arrive to the desired bound for $\operatorname{Prob}^{N}(B)$

$\operatorname{Prob}^{N}(B) \leq \sum_{I \in \mathcal{I}} \operatorname{Prob}^{N}\left(B_{I} \cap \Delta_{I}^{N}\right)<\left(\begin{array}{c}N \\ n_{\theta}\end{array}\right)(1-\epsilon)^{N-n_{\theta}}$.

The last part of the proof is nothing but algebraic manipulations on bound (11) to show that, if $N$ is chosen according to (2), then

$$
\left(\begin{array}{l}
N \\
n_{\theta}
\end{array}\right)(1-\epsilon)^{N-n_{\theta}} \leq \beta
$$

so concluding the proof. These manipulations are reported next. Any of the following inequality implies the next in a top-down fashion, where the first one is (2):

$$
\begin{aligned}
N \geq & \frac{1}{1-\nu}\left(\frac{1}{\epsilon} \ln \frac{1}{\beta}+n_{\theta}+\right. \\
& \left.+\frac{n_{\theta}}{\epsilon} \ln \frac{1}{\nu \epsilon}+\frac{1}{\epsilon} \ln \left(\left(\frac{n_{\theta}}{e}\right)^{n_{\theta}} \frac{1}{n_{\theta} !}\right)\right) \\
(1-\nu) N \geq & \frac{1}{\epsilon} \ln \frac{1}{\beta}+n_{\theta}+\frac{n_{\theta}}{\epsilon} \ln \frac{1}{\nu \epsilon}+\frac{1}{\epsilon} \ln \left(\left(\frac{n_{\theta}}{e}\right)^{n_{\theta}} \frac{1}{n_{\theta} !}\right) \\
(1-\nu) N \geq & \frac{1}{\epsilon} \ln \frac{1}{\beta}+n_{\theta}+\frac{n_{\theta}}{\epsilon}\left(\ln \frac{n_{\theta}}{\nu \epsilon}-1\right)-\frac{1}{\epsilon} \ln \left(n_{\theta} !\right) \\
N \geq & \frac{1}{\epsilon} \ln \frac{1}{\beta}+n_{\theta}+\frac{n_{\theta}}{\epsilon}\left(\ln \frac{n_{\theta}}{\nu \epsilon}-1+\frac{\nu N \epsilon}{n_{\theta}}\right)-\frac{1}{\epsilon} \ln \left(n_{\theta} !\right) \\
N \geq & \frac{1}{\epsilon} \ln \frac{1}{\beta}+n_{\theta}+\frac{n_{\theta}}{\epsilon} \ln N-\frac{1}{\epsilon} \ln \left(n_{\theta} !\right)
\end{aligned}
$$

where the last implication can be justified by observing that $\ln x \geq 1-1 / x$, for $x>0$, and applying this inequality with

$$
\operatorname{Prob}^{N-n_{\theta}}\left\{\left(\delta^{\left(n_{\theta}+1\right)}, \ldots, \delta^{(N)}\right):\left(\bar{\delta}^{(1)}, \ldots, \bar{\delta}^{\left(n_{\theta}\right)}, \delta^{\left(n_{\theta}+1\right)}, \ldots, \delta^{(N)}\right) \in B_{\left\{1, \ldots, n_{\theta}\right\}} \cap \Delta_{\left\{1, \ldots, n_{\theta}\right\}}^{N}\right\}<(1-\epsilon)^{N-n_{\theta}}
$$


$x=n_{\theta} / \nu N \epsilon$. Proceeding from (13), the next inequalities in the chain are

$$
\begin{aligned}
\ln \beta & \geq-\epsilon N+\epsilon n_{\theta}+n_{\theta} \ln N-\ln \left(n_{\theta} !\right) \\
\beta & \geq \frac{N^{n_{\theta}}}{n_{\theta} !} e^{-\epsilon\left(N-n_{\theta}\right)} \\
\beta & \geq \frac{N(N-1) \cdots\left(N-n_{\theta}+1\right)}{n_{\theta} !}(1-\epsilon)^{N-n_{\theta}}
\end{aligned}
$$

where, in the last implication, we have used the fact that $e^{-\epsilon\left(N-n_{\theta}\right)} \geq(1-\epsilon)^{N-n_{\theta}}$, as it follows by taking logarithm of the two sides and further noting that $-\epsilon \geq \ln (1-\epsilon)$. The last inequality can be rewritten as

$$
\beta \geq\left(\begin{array}{c}
N \\
n_{\theta}
\end{array}\right)(1-\epsilon)^{N-n_{\theta}}
$$

which is (12).

So far, we have assumed that $\mathrm{RCP}_{N}$ is feasible for any selection of $\delta^{(1)}, \ldots, \delta^{(N)}$. Relax now this assumption and call $F \subseteq \Delta^{N}$ the set where $\mathrm{RCP}_{N}$ is indeed feasible. The same derivation can then be worked out in the domain $F$, instead of $\Delta^{N}$, leading to the conclusion that (11) holds with $B \doteq\left\{\left(\delta^{(1)}, \ldots, \delta^{(N)}\right) \in F: V\left(\hat{\theta}_{N}\right)>\epsilon\right\}$. This concludes the proof.

\section{APPLICATIONS to Robust CONTROL}

In this section, we discuss a few relevant control analysis and synthesis problems that can be naturally cast in the robust convex program format, and for which no deterministic polynomial-time algorithm is known that computes an exact solution. For these problems, the solution approach that we propose is to first relax the problem in a probabilistic sense and then solve the probabilistic problem via the randomized scenario approach presented in the previous sections.

\section{A. Analysis and Synthesis via Parameter-Dependent Lyapunov Functions}

Consider the family of linear systems described in state-space form as

$$
\dot{x}=A(\delta) x, \quad \delta \in \Delta
$$

where $x \in \mathbb{R}^{n_{x}}$ is the state variable, and the parameter $\delta \in \Delta \subseteq$ $\mathbb{R}^{n_{\delta}}$ parameterizing the system family is unknown, but constant in time. In the sequel, we will refer to system families of the type (14) simply as "uncertain systems."

Let $P(\xi, \delta)$ be a family of symmetric matrices depending on a design parameter $\xi \in \mathbb{R}^{n_{\xi}}$ and on the uncertainty parameter $\delta \in \Delta$, and assume that $P(\xi, \delta)$ is linear in $\xi$, for all $\delta \in \Delta$. The dependence of $P(\xi, \delta)$ on the uncertainty $\delta$, as well as the dependence of $A(\delta)$ on $\delta$, are instead left generic. We introduce the following sufficient condition for robust stability, which follows directly from the standard Lyapunov theory.

Definition 5 (Generalized Quadratic Stability $G Q S)$ : Given a symmetric matrix function $P(\xi, \delta)$, linear in $\xi \in \mathbb{R}^{n_{\xi}}$ for all $\delta \in \Delta$, the uncertain system (14) is said to be quadratically stable with respect to $P(\xi, \delta)$ if there exists $\xi \in \mathbb{R}^{n_{\xi}}$ such that

$$
\left[\begin{array}{cc}
-P(\xi, \delta) & 0 \\
0 & A^{T}(\delta) P(\xi, \delta)+P(\xi, \delta) A(\delta)
\end{array}\right] \prec 0, \quad \forall \delta \in \Delta
$$

( $\prec$ means negative definite). Such a $P(\xi, \delta)$ is called a Lyapunov matrix for the uncertain system (14).

For specific choices of the parameterization $P(\xi, \delta)$, the above GQS criterion clearly encompasses the popular quadratic stability (QS, [11], [12]) and affine quadratic stability (AQS, [26]) criteria, as well as the biquadratic stability condition of [45]. For instance, the quadratic stability condition is recovered by choosing $P(\xi, \delta)=P$ (i.e., $\xi$ contains the free elements of $P=P^{T}$, and there is no dependence on $\delta$ ), which amounts to determining a single Lyapunov matrix $P$ that simultaneously satisfies (15). The AQS condition is instead obtained by choosing

$$
P(\xi, \delta)=P_{0}+\delta_{1} P_{1}+\cdots+\delta_{n_{\delta}} P_{n_{\delta}}
$$

where $\xi$ represents the free elements in the matrices $P_{i}=P_{i}^{T}$, $i=0, \ldots, n_{\delta}$. Notice that QS, AQS and GQS constitute a hierarchy of sufficient conditions for robust stability having decreasing conservatism. However, even the simplest (and most conservative) QS condition is hard to check numerically. Only in the case when the set $\{A(\delta), \delta \in \Delta\}$ is a polytope, the QS condition is exactly checkable numerically via convex optimization, [11], [12]. As a matter of fact, in this case a classical vertex result holds which permits to convert the infinite number of constraints entailed by (15) into a finite number of LMIs involving the vertices of the polytope. Notice however that in the classical case when $A(\delta)$ is an interval matrix, the number of vertices of the polytope grows as $2^{n_{x}^{2}}$, which means that QS cannot be checked with a computational effort that is polynomial in the problem size $n_{x}$.

Now, notice that a key feature of the condition (15) is that, for any fixed $\delta \in \Delta$ it represents a convex LMI condition in $\xi$, and therefore finding a feasible parameter $\xi$ amounts indeed to solving a robust convex program. This is the key observation that makes the scenario paradigm well-suited for probabilistic analysis within the context of generalized quadratic stability. With pre-specified confidence, a matrix $P(\xi, \delta)$ generated by a scenario solution would be a Lyapunov matrix for all but a small fraction of the systems in the family (14).

1) Formalization as $\mathrm{RCP}_{N}$ : Notice that condition (15) is a feasibility condition expressed by a strict matrix inequality, while the robust convex problem considered in previous sections is a minimization problem subject to a nonstrict inequality condition (we have $f(\theta, \delta) \leq 0$ as opposed to $f(\theta, \delta)<0$ ). The precise formalization of the GQS problem within the scenario setting can be done in more than one way and it is to a certain extent a matter of taste. Here, as an illustration, we further develop this first example to indicate a possible way to cast it within the setup of Section III. It is tacitly understood that similar formalizations apply to all other examples. 
First, set an optimization problem with the format of a robust convex program as follows:

$$
\begin{aligned}
& \text { RCP : } \min \alpha \quad \text { subject to : } \\
& -I \preceq\left[\begin{array}{cc}
-P(\xi, \delta) & 0 \\
0 & A^{T}(\delta) P(\xi, \delta)+P(\xi, \delta) A(\delta)
\end{array}\right] \preceq \alpha I \\
& \quad \forall \delta \in \Delta .
\end{aligned}
$$

Then, assume a probability measure Prob over the uncertainties is given, and build the scenario counterpart of the problem

$\operatorname{RCP}_{N}: \min \alpha$ subject to :

$$
\begin{aligned}
-I & \preceq\left[\begin{array}{cc}
-P\left(\xi, \delta^{(i)}\right) & 0 \\
0 & A^{T}\left(\delta^{(i)}\right) P\left(\xi, \delta^{(i)}\right)+P\left(\xi, \delta^{(i)}\right) A\left(\delta^{(i)}\right)
\end{array}\right] \\
& \preceq \alpha I, \quad i=1, \ldots, N
\end{aligned}
$$

where the scenarios $\delta^{(i)}$ are independently extracted at random according to Prob. Here, the optimization variable is $\theta \doteq(\xi, \alpha)$. Note also that the lower bound $-I$ has been introduced without loss of generality since, otherwise, the solution may escape to infinity due to homogeneity of the constraint.

Applying Theorem 1 we can then conclude that, with probability at least $1-\beta$, either $\mathrm{RCP}_{N}$ is unfeasible, so that RCP and the original GQS is unfeasible, or the solution $(\bar{\xi}, \bar{\alpha})$ of $\mathrm{RCP}_{N}$ is a $\epsilon$-level solution for RCP. In the latter case, if $\bar{\alpha} \geq 0$, it is easily seen that GQS is again unfeasible. Finally, if $\bar{\alpha}<0$, then $P(\bar{\xi}, \delta)$ is a $\epsilon$-level solution for GQS. Applicability of Theorem 1 subsumes that Assumption 2 holds, while in general situations one can resort to Theorem 4 in Appendix A.

\section{B. State-Feedback Stabilization}

Consider the uncertain system

$$
\dot{x}=A(\delta) x+B(\delta) u
$$

where $x \in \mathbb{R}^{n_{x}}$ is the state variable, $u \in \mathbb{R}^{n_{u}}$ is the control input, and matrices $A$ and $B$ are generic functions of $\delta \in \Delta$.

Suppose we want to stabilize (17) by means of a state-feedback control law $u=K x$, where $K \in \mathbb{R}^{n_{u}, n_{x}}$ is a static feedback gain. The resulting closed-loop system is robustly stable if and only if $A_{c l}(\delta) \doteq A(\delta)+B(\delta) K$ is Hurwitz for all $\delta \in \Delta$.

Using the enhanced LMI characterization proposed in [2] (Theorem 3.1), robust stabilizability of (17) is equivalent to the existence of matrices $V \in \mathbb{R}^{n_{x}, n_{x}}, R \in \mathbb{R}^{n_{u}, n_{x}}$, and a Lyapunov symmetric matrix function $P(\delta) \in \mathbb{R}^{n_{x}, n_{x}}$ such that

$$
\left[\begin{array}{ccc}
-\left(V+V^{T}\right) & V^{T} A^{T}(\delta)+R^{T} B^{T}(\delta)+P(\delta) & V^{T} \\
* & -P(\delta) & 0 \\
* & * & -P(\delta)
\end{array}\right] \prec 0
$$

(asterisks denote entries that are easily inferred from symmetry). If a feasible solution is found, the robustly stabilizing feedback gain is recovered as $K=R V^{-1}$. A sufficient condition for robust stabilizability is hence readily obtained by considering a specific parameterized matrix function family $P(\xi, \delta)$ (linear in the parameter $\xi$, for any fixed $\delta \in \Delta$ ) in the above condition. The resulting problem is convex in the decision variable $\theta \doteq$ $(\xi, V, R)$, for any fixed $\delta \in \Delta$, and it is therefore a robust convex problem. Notice again that this robust problem is hard to solve in general. As an exception, in the special case when $[A(\delta) B(\delta)]$ is affine in $\delta, \Delta$ is a hypercube, and $P(\delta)$ is chosen in the affine form (16), the previous robust condition can be transformed by a standard "vertexization" argument into a finite set of LMIs involving the vertex matrices, and hence solved exactly (this latter special case is indeed the one presented in [2]). We remark however again that the number of vertices (and, hence, of LMI constraints) grows exponentially with the number of uncertain parameters $n_{\delta}$, which makes this standard approach practically unviable in cases when $n_{\delta}$ is large.

This robust state-feedback stabilization problem is amenable to the scenario randomization approach similarly to the problem in Section V-A. A numerical example is presented in Section V-D.

\section{Other Problems}

In the previous sections, only a few control problems amenable to the scenario reformulation have been illustrated. This is just a sample of possible problems and many more can be considered, such as robust $H_{\infty}$ or $H_{2}$ state feedback synthesis (see e.g., [2]), linear parameter-varying (LPV) design (see, e.g., [5] for a formulation of the LPV synthesis problem in the form of a robust convex semidefinite program, and [24] for a randomized solution approach, alternative to the one proposed in this paper), LP-based robust pole assignment (see [32]), the various robust design problems based on parameter-dependent linear matrix inequalities presented in [1], as well as problems in the robust model predictive control setup. For discrete-time systems, the robust analysis and design criteria proposed in [21] and [22] are also directly suitable for the scenario technique. Also, problems of set-membership state reachability and filtering [14], [18] may be efficiently solved in a probabilistic sense by the proposed methodology.

In the next section, we present a numerical example of the scenario design approach.

\section{A Numerical Example}

Consider a robust state-feedback stabilization problem of the form presented in Section V-B. In particular, let the uncertain system be given by

$$
\dot{x}=A(\delta) x+B u
$$

where

$$
B=\left[\begin{array}{llll}
1 & 0 & 1 & 1 \\
0 & 1 & 0 & 1
\end{array}\right]^{T}
$$

and $A(\delta)$ is as given at the bottom of the next page, with $\Omega \doteq$

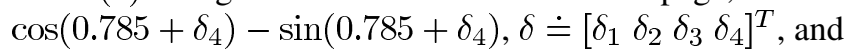

$$
\Delta=\left\{\delta:\left|\delta_{1}\right| \leq 0.2,\left|\delta_{2}\right| \leq 0.2,\left|\delta_{3}\right| \leq 0.2,\left|\delta_{4}\right| \leq 0.2\right\} .
$$

The objective is to determine a state-feedback control law $u=K x$, such that the resulting closed-loop system is robustly stable.

Using quadratic stability as a sufficient condition for robust stability, from (18) we have that such controller exists if there 
exist $V \in \mathbb{R}^{4,4}, R \in \mathbb{R}^{2,4}$, and a Lyapunov matrix $P=P^{T}$ such that (18) is satisfied. If a feasible solution is found, the feedback gain is recovered as $K=R V^{-1}$.

Assuming uniform distribution over $\Delta$, and proceeding as discussed in Section V-A.II, we write the scenario version of the problem as

$$
\begin{aligned}
& \min \alpha \text { subject to : } \\
& -I \preceq\left[\begin{array}{ccc}
-\left(V+V^{T}\right) & V^{T} A^{T}\left(\delta^{(i)}\right)+R^{T} B^{T}+P & V^{T} \\
* & -P & 0 \\
* & * & -P
\end{array}\right] \preceq \alpha I, \\
& \quad i=1, \ldots, N
\end{aligned}
$$

where $\delta^{(i)}$ are independent samples uniformly extracted from $\Delta$.

The design variable in this problem is $\theta \doteq(V, R, P, \alpha)$, which contains $n_{\theta}=16+8+10+1=35$ free variables. Hence, fixing a-priori probabilistic levels $\epsilon=0.1$ and $\beta=0.01$, and using bound (2), we determine the sample size $N \geq 1780$.

The numerical solution of one instance of the above scenario problem yielded optimal objective $\alpha=-0.0635$, corresponding to the controller

$$
K=\left[\begin{array}{cccc}
-3.8877 & 1.8740 & -2.6492 & -1.6638 \\
2.5376 & -1.5973 & 2.4038 & -4.0906
\end{array}\right]
$$

Next, we run an a-posteriori Monte-Carlo analysis of robust stability. We can use a much larger sample size for this a-posteriori analysis, since no numerical optimization is involved in the process. This a-posteriori test is conducted by testing directly whether $A(\delta)+B K$ is Hurwitz for a very large number $\left(6 \times 10^{6}\right)$ of sampled $\delta$ 's. Interestingly, for the computed controller $K$, the a-posteriori estimated probability of instability of $A(\delta)+B K$ resulted to be equal to zero, which, using Hoeffding's inequality [30], means for instance that we can claim with confidence greater than $99.999 \%$ that our controller may fail at most on a set of $\delta$ 's having volume 0.001 .

\section{CONCLUSION}

This paper presented a novel approach to robust control design based on the concept of uncertainty scenarios. Within this framework, if the robustness requirements are imposed in a probabilistic sense, then a wide class of control analysis and synthesis problems are amenable to efficient numerical solution. This solution is computed solving a convex optimization problem having a finite number $N$ of sampled constraints. The main contribution of the paper is to provide an explicit and efficient bound on the number of scenarios required to obtain a design that guarantees an a-priori specified probabilistic robustness level.
This methodology is illustrated by control design examples that present difficulties when tackled by means of standard worst-case techniques. We believe that, due to its intrinsic simplicity, the scenario approach will be an appealing solution technique for many practical engineering design problems, also beyond the control applications mentioned in this paper.

\section{APPENDIX A \\ RELEASING THE UNIQUENESS ASSUMPTION}

In Section III, the theory has been developed under Assumption 2 requiring that $\mathrm{RCP}_{N}$ is either unfeasible or, if feasible, it admits a unique optimal solution. Here, we drop Assumption 2 and consider the general case allowing for nonuniqueness of the solution or nonexistence of the solution even when $\mathrm{RCP}_{N}$ is feasible (i.e., the solution escapes to infinity).

\section{A. Nonuniqueness of the Solution}

We follow the same approach as in [17, Sec. 4.1]. Suppose that when problem $\operatorname{RCP}_{N}$ admits more than one optimal solution we break the tie by a tie-break rule as follows:

Tie-Break Rule: Let $t_{i}(\theta), i=1, \ldots, p$, be given convex and continuous functions. Among the optimal solutions for $\mathrm{RCP}_{N}$, select the one that minimizes $t_{1}(\theta)$. If indetermination still occurs, select among the $\theta$ that minimize $t_{1}(\theta)$ the solution that minimizes $t_{2}(\theta)$, and so on with $t_{3}(\theta), t_{4}(\theta), \ldots$ We assume that functions $t_{i}(\theta), i=1, \ldots, p$, are selected so that the tie is broken within $p$ steps at most. As a simple example of a tie-break rule, one can consider $t_{1}(\theta)=\theta_{1}, t_{2}(\theta)=\theta_{2}, \ldots$

From now on, by "optimal solution," we mean either the unique optimal solution, or the solution selected according to the Tie-break rule, if multiple optimal solutions occur.

Theorem 1 holds unchanged if we drop the uniqueness requirement in Assumption 2, provided that "optimal solution" is intended in the indicated sense.

To see this, generalize Definition 4 of support constraints to: The kth constraint $\mathcal{X}_{k}$ is a support constraint for $\mathcal{P}$ if problem $\mathcal{P}_{k}$ has an optimal solution $x_{k}^{*}$ such that $x_{k}^{*} \neq x^{*}$. Indeed this definition generalizes Definition 4 since, in case of a single optimal solution (single without applying the Tie-break rule), $x_{k}^{*} \neq x^{*}$ is equivalent to $J_{k}^{*}<J^{*}$. In [17, Sec. 4.1], it is proven that Theorem 3 holds true with this extended definition of support constraint (i.e., the number of support constraints is at most $n_{\theta}$ ), and then an inspection of the proof of Theorem 1 in Section IV reveals that this proof goes through unaltered in the present setting, so concluding that Theorem 1 still holds.

\section{B. Nonexistence of the Solution}

Even when $\mathrm{RCP}_{N}$ is feasible, it may happen that no optimal solution exists since the set for $\theta$ allowed by the extracted con-

$$
A(\delta)=\left[\begin{array}{cccc}
2\left(1+\delta_{3}\right) \Omega & -2-2 \delta_{1}-2\left(1+\delta_{3}\right) \Omega & 2\left(1+\delta_{3}\right) \sin \left(0.785+\delta_{4}\right) & 2+2 \delta_{1}+2\left(1+\delta_{3}\right) \Omega \\
0 & -2-2 \delta_{1} & 0 & 4+2 \delta_{1}+2 \delta_{2} \\
4\left(1+\delta_{3}\right) \cos \left(0.785+\delta_{4}\right) & -4\left(1+\delta_{3}\right) \cos \left(0.785+\delta_{4}\right) & -2\left(1+\delta_{3}\right) \Omega & 4\left(1+\delta_{3}\right) \Omega \\
0 & 0 & 0 & 2+2 \delta_{2}
\end{array}\right]
$$


straints is unbounded in such a way that the optimal solution escapes to infinity. In this section, we further generalize Theorem 1 so as to cope with this situation too and then provide a reformulation of Theorem 1 (Theorem 4 ) that covers all possible situations.

Suppose that a random extraction of a multisample $\delta^{(1)}, \ldots, \delta^{(N)}$ is rejected when the problem is feasible but no optimal solution exists, and another extraction is performed in this case. Then, the result of Theorem 1 holds if attention is restricted to the accepted multisamples. This idea is now formalized.

Let $D \subseteq \Delta^{N}$ be the set where $\mathrm{RCP}_{N}$ is feasible but an optimal solution does not exist (it escapes to infinity) and assume that its complement $A=D^{c}$ has positive probability: $\operatorname{Prob}^{N}(A)>0$. Moreover, let $\operatorname{Prob}_{A}^{N}$ be the probability $\operatorname{Prob}^{N}$ restricted to $A: \operatorname{Prob}_{A}^{N}(\cdot) \doteq \operatorname{Prob}^{N}(\cdot \cap A) / \operatorname{Prob}^{N}(A)$. In addition, assume that if a problem with, say, $m$ constraints is feasible and admits optimal solution, then, after adding an extra $(m+1)$ th constraint, if the problem remains feasible, then an optimal solution continues to exists (this rules out the possibility of pathological situations where adding a constraint forces the solution to drift away to infinity).

Going trough the proof of Theorem 1, we can readily see that it remains valid if attention is restricted to $A$. Precisely, the following theorem holds.

Theorem 4: Fix two real numbers $\epsilon \in(0,1)$ (level parameter) and $\beta \in(0,1)$ (confidence parameter). If

$$
\begin{aligned}
N & \geq N_{\text {gen }}(\epsilon, \beta) \\
& \left.\doteq \inf _{\nu \in(0,1)} \frac{1}{1-\nu}\left(\frac{1}{\epsilon} \ln \frac{1}{\beta}+n_{\theta}+\frac{n_{\theta}}{\epsilon} \ln \frac{1}{\nu \epsilon}+\frac{1}{\epsilon} \ln \frac{\left(\frac{n_{\theta}}{e}\right)^{n_{\theta}}}{n_{\theta} !}\right)\right]
\end{aligned}
$$

then, with probability $\operatorname{Prob}_{A}^{N}$ no smaller than $1-\beta$, either the scenario problem $\mathrm{RCP}_{N}$ is unfeasible, and hence also the initial robust convex program is unfeasible; or, $\mathrm{RCP}_{N}$ is feasible, and then its optimal solution $\hat{\theta}_{N}$ (unique after the tie-break rule has been applied) is $\epsilon$-level robustly feasible.

The proof of this theorem is obtained by following the same steps as in the proof of Theorem 1 in Section IV with a few simple amendments, as sketched in the following.

Similarly to the proof of Theorem 1, forget for the time being that $\mathrm{RCP}_{N}$ can be unfeasible and assume $F=\Delta^{N}$. In the present context, interpret all subset of $\Delta^{N}$ (e.g., $\Delta_{I}^{N}, B, B_{I}$ ) as subsets of $A$, so e.g., $\Delta_{I}^{N} \doteq\left\{\left(\delta^{(1)}, \ldots, \delta^{(N)}\right) \in A: \hat{\theta}_{I}=\hat{\theta}_{N}\right\}$. Everything in the proof goes through unaltered till (10). In (10), drop the last step and consider the inequality

$$
\begin{aligned}
\operatorname{Prob}^{N}( & \left.B_{\left\{1, \ldots, n_{\theta}\right\}} \cap \Delta_{\left\{1, \ldots, n_{\theta}\right\}}^{N}\right) \\
& <(1-\epsilon)^{N-n_{\theta}} \cdot \operatorname{Prob}^{n_{\theta}}\left(\text { base of } B_{\left\{1, \ldots, n_{\theta}\right\}}\right) .
\end{aligned}
$$

Now, the cylinder $B_{\left\{1, \ldots, n_{\theta}\right\}}$ does not intersect $D$ since any multisample in the cylinder is formed by the first $n_{\theta}$ samples that generate the optimal solution $\hat{\theta}_{\left\{1, \ldots, n_{\theta}\right\}}$, plus the remaining
$N-n_{\theta}$ samples that, in conjunction with the first $n_{\theta}$, either make the problem unfeasible, or, if feasible, add constraints so still preventing escape to infinity. So

$$
\begin{aligned}
\operatorname{Prob}^{n_{\theta}}\left(\text { base of } B_{\left\{1, \ldots, n_{\theta}\right\}}\right) & =\operatorname{Prob}^{N}\left(B_{\left\{1, \ldots, n_{\theta}\right\}}\right) \\
\leq 1-\operatorname{Prob}^{N}(D) & =\operatorname{Prob}^{N}(A)
\end{aligned}
$$

which, used in (19) gives

$$
\operatorname{Prob}^{N}\left(B_{\left\{1, \ldots, n_{\theta}\right\}} \cap \Delta_{\left\{1, \ldots, n_{\theta}\right\}}^{N}\right)<(1-\epsilon)^{N-n_{\theta}} \cdot \operatorname{Prob}^{N}(A)
$$

and, after substitution in (11), we obtain

$$
\begin{aligned}
\operatorname{Prob}^{N}(B) & \leq \sum_{I \in \mathcal{I}} \operatorname{Prob}^{N}\left(B_{I} \cap \Delta_{I}\right) \\
& <\left(\begin{array}{c}
N \\
n_{\theta}
\end{array}\right)(1-\epsilon)^{N-n_{\theta}} \cdot \operatorname{Prob}^{N}(A) \leq \beta \cdot \operatorname{Prob}^{N}(A)
\end{aligned}
$$

or, equivalently

$$
\frac{\operatorname{Prob}^{N}(B)}{\operatorname{Prob}^{N}(A)} \leq \beta
$$

Since the left-hand side is $\operatorname{Prob}_{A}^{N}(B)$, the desired result remains proven. The case when $F$ is a strict subset of $\Delta^{N}$ can be dealt with without any additional complication.

\section{APPENDIX B \\ PROOF OF THEOREM 2}

The first claim is immediate, since from Theorem 1, with probability at least $1-\beta$, if $\mathrm{RCP}_{N}$ is feasible, then its optimal solution $\hat{\theta}_{N}$ satisfies $V\left(\hat{\theta}_{N}\right) \leq \epsilon$, i.e., it is a feasible, albeit possibly not optimal, solution for problem $\operatorname{CCP}(\epsilon)$, and hence $J_{\mathrm{RCP}_{N}} \geq J_{\mathrm{CCP}(\epsilon)}$.

To prove the second claim, notice that if $\theta$ is feasible for problem $\operatorname{CCP}\left(\epsilon_{1}\right)$ with $\epsilon_{1}=1-(1-\beta)^{1 / N}$, i.e.,

$$
\operatorname{Prob}\{\delta \in \Delta: f(\theta, \delta)>0\} \leq 1-(1-\beta)^{1 / N}
$$

then for each of $N$ independent extractions $\delta^{(1)}, \ldots, \delta^{(N)}$ of $\delta$ it holds that

$$
\operatorname{Prob}\left\{\delta^{(i)} \in \Delta: f\left(\theta, \delta^{(i)}\right) \leq 0\right\} \geq(1-\beta)^{1 / N}
$$

for $i=1, \ldots, N$ and, hence, by independence, the joint event $\left\{\left(\delta^{(1)}, \ldots, \delta^{(N)}\right) \in \Delta^{N}: f\left(\theta, \delta^{(i)}\right) \leq 0, i=1, \ldots, N\right\}$ holds with probability at least $1-\beta$. This means that, with probability at least $1-\beta$, a feasible point for $\operatorname{CCP}\left(\epsilon_{1}\right)$ is also a feasible point for $\mathrm{RCP}_{N}$. We now have two possibilities, depending on whether $\operatorname{CCP}\left(\epsilon_{1}\right)$ attains an optimal solution (i.e., a $\hat{\theta}$ feasible for $\operatorname{CCP}\left(\epsilon_{1}\right)$ exists such that $\left.c^{T} \hat{\theta}=J_{\mathrm{CCP}\left(\epsilon_{1}\right)}\right)$ or not. In the first situation ( $\hat{\theta}$ exists), taking $\theta=\hat{\theta}$ in the previous reasoning immediately implies that $J_{\mathrm{RCP}_{N}} \leq J_{\mathrm{CCP}}\left(\epsilon_{1}\right)$, as desired.

In the second situation ( $\hat{\theta}$ does not exist), consider a point $\bar{\theta}$ which is feasible for $\mathrm{CCP}\left(\epsilon_{1}\right)$ and such that $c^{T} \bar{\theta} \leq J_{\mathrm{CCP}\left(\epsilon_{1}\right)}+$ $\rho$, for some $\rho>0$ (such a $\bar{\theta}$ exists since $J_{\mathrm{CCP}\left(\epsilon_{1}\right)}=\inf c^{T} \theta$ over $\theta$ 's that are feasible for $\left.\operatorname{CCP}\left(\epsilon_{1}\right)\right)$. By the previous reasoning, 
this implies that, with probability at least $1-\beta$, the point $\bar{\theta}$ is also feasible for problem $\mathrm{RCP}_{N}$, entailing

$$
\begin{array}{r}
\operatorname{Prob}\left\{\left(\delta^{(1)}, \ldots, \delta^{(N)}\right) \in \Delta^{N}: J_{\mathrm{RCP}_{N}} \leq J_{\mathrm{CCP}\left(\epsilon_{1}\right)}+\rho\right\} \\
\geq 1-\beta .
\end{array}
$$

For the purpose of contradiction, suppose now that result 2 in the theorem is violated so that $J_{\mathrm{RCP}_{N}}>J_{\mathrm{CCP}\left(\epsilon_{1}\right)}$ with probability larger than $\beta$. Since

$$
\begin{aligned}
& \left\{\left(\delta^{(1)}, \ldots, \delta^{(N)}\right) \in \Delta^{N}: J_{\mathrm{RCP}_{N}}>J_{\mathrm{CCP}\left(\epsilon_{1}\right)}\right\} \\
& \quad=\bigcup_{\nu>0}\left\{\left(\delta^{(1)}, \ldots, \delta^{(N)}\right) \in \Delta^{N}: J_{\mathrm{CCP}_{N}}>J_{\mathrm{CCP}\left(\epsilon_{1}\right)}+\frac{1}{\nu}\right\}
\end{aligned}
$$

then

$$
\begin{aligned}
& \beta<\operatorname{Prob}^{N}\left\{\left(\delta^{(1)}, \ldots, \delta^{(N)}\right) \in \Delta^{N}: J_{\mathrm{RCP}_{N}}>J_{\mathrm{CCP}\left(\epsilon_{1}\right)}\right\} \\
& =\lim _{\nu \rightarrow \infty} \operatorname{Prob}^{N}\left\{\left(\delta^{(1)}, \ldots, \delta^{(N)}\right) \in \Delta^{N}: J_{\mathrm{RCP}_{N}}>J_{\mathrm{CCP}\left(\epsilon_{1}\right)}+\frac{1}{\nu}\right\}
\end{aligned}
$$

and we conclude that there exists a $\bar{\nu}$ such that $J_{\mathrm{RCP}_{N}}>$ $J_{\mathrm{CCP}\left(\epsilon_{1}\right)}+1 / \bar{\nu}$ with probability larger than $\beta$. However, this contradicts (20) for $\rho=1 / \bar{\nu}$, so concluding the proof.

\section{REFERENCES}

[1] P. Apkarian and H. D. Tuan, "Parameterized LMI's in control theory," SIAM J. Control Optim., vol. 38, no. 4, pp. 1241-1264, 2000.

[2] P. Apkarian, H. D. Tuan, and J. Bernussou, "Continuous-time analysis, eigenstructure assignment, and $\mathrm{H}_{2}$ synthesis with enhanced linear matrix inequalities (LMI) characterizations," IEEE Trans. Autom. Control, vol. 46, no. 12, pp. 1941-1946, Dec. 2001.

[3] B. R. Barmish and C. M. Lagoa, "The uniform distribution: A rigorous justification for its use in robustness analysis," Math. Control, Signals, Syst., vol. 10, pp. 203-222, 1997.

[4] B. R. Barmish and P. S. Scherbakov, "On avoiding vertexization of robustness problems: The approximate feasibility concept," in Proc. IEEE Conf. Decision and Control, vol. 2, Sydney, Australia, Dec. 2000, pp. 1031-1036.

[5] G. Becker and A. Packard, "Robust performance of linear parametrically varying systems using parametrically-dependent linear feedback," Syst. Control Lett., vol. 23, pp. 205-215, 1994.

[6] A. Ben-Tal and A. Nemirovski, "Robust truss topology design via semidefinite programming," SIAM J. Optim., vol. 7, no. 4, pp. 991-1016, 1997.

[7] —, "Robust convex optimization," Math. Oper. Res., vol. 23, no. 4, pp. 769-805, 1998.

[8] — , "Robust solutions of uncertain linear programs," Oper. Res. Lett., vol. 25 , no. 1 , pp. $1-13,1999$.

[9] _ "On tractable approximations of uncertain linear matrix inequalities affected by interval uncertainty," SIAM J. Optim., vol. 12, no. 3, pp. $811-833,2002$.

[10] V. D. Blondel and J. N. Tsitsiklis, "A survey of computational complexity results in systems and control," Automatica, vol. 36, pp. 1249-1274, 2000.

[11] S. Boyd, L. El Ghaoui, E. Feron, and V. Balakrishnan, Linear Matrix Inequalities in System and Control Theory. Philadelphia, PA: SIAM, 1994, vol. 15, Studies in Applied Mathematics.

[12] S. Boyd and Q. Yang, "Structured and simultaneous Lyapunov functions for system stability problems," Int. J. Control, vol. 49, pp. 2215-2240, 1989.

[13] R. P. Braatz, P. M. Young, J. C. Doyle, and M. Morari, "Computational complexity of $\mu$ calculation," IEEE Trans. Autom. Control, vol. 39, no. 5, pp. 1000-1002, May 1994.

[14] G. Calafiore, "Set simulations for quadratic systems," IEEE Trans. Autom. Control, vol. 48, no. 5, pp. 800-805, May 2003.

[15] G. Calafiore and M. C. Campi, "Interval predictors for unknown dynamical systems: An assessment of reliability," in Proc. IEEE Conf. Decision and Control, Las Vegas, NV, Dec. 2002, pp. 4766-4779.
[16] — "A learning theory approach to the construction of predictor models," AIMS J. Discrete Continuous Dyna. Syst., pp. 156-166, 2003.

[17] _ - "Uncertain convex programs: Randomized solutions and confidence levels," Math. Program., vol. 102, no. 1, pp. 25-46, 2005.

[18] G. Calafiore and L. El Ghaoui, "Ellipsoidal bounds for uncertain linear equations and dynamical systems," Automatica, vol. 50, no. 5, pp. 773-787, 2004.

[19] G. Calafiore and B. T. Polyak, "Fast algorithms for exact and approximate feasibility of robust LMIs," IEEE Trans. Autom. Control, vol. 46, no. 11, pp. 1755-1759, Nov. 2001.

[20] G. Calafiore, F. Dabbene, and R. Tempo, "Randomized algorithms for probabilistic robustness with real and complex structured uncertainty," IEEE Trans. Autom. Control, vol. 45, no. 12, pp. 2218-2235, Dec. 2000.

[21] M. C. de Oliveira, J. Bernussou, and J. C. Geromel, "A new discretetime robust stability condition," Syst. Control Lett., vol. 37, pp. 261-265, 1999.

[22] M. C. de Oliveira, J. C. Geromel, and J. Bernussou, "Extended $H_{2}$ and $H_{\infty}$ norm characterization and controller parameterization for discretetime systems," Int. J. Control, vol. 75, no. 9, pp. 666-679, 2002.

[23] S. Floyd and M. Warmuth, "Sample compression, learnability, and the Vapnik-Chervonenkis dimension," Mach. Learn., pp. 1-36, 1995.

[24] Y. Fujisaki, F. Dabbene, and R. Tempo, "Probabilistic design of LPV control systems," Automatica, vol. 39, no. 8, pp. 1323-1337, 2003.

[25] P. Gahinet, "Explicit controller formulas for LMI-based $H_{\infty}$ synthesis," Automatica, vol. 32, no. 7, pp. 1007-1014, 1996.

[26] P. Gahinet, P. Apkarian, and M. Chilali, "Affine parameter-dependent Lyapunov functions and real parametric uncertainty," IEEE Trans. Autom. Control, vol. 41, no. 3, pp. 436-442, Mar. 1996.

[27] L. El Ghaoui and H. Lebret, "Robust solutions to least-squares problems with uncertain data," SIAM J. Matrix Anal. Appl., vol. 18, no. 4, pp. 1035-1064, 1997.

[28] — - "Robust solutions to uncertain semidefinite programs," SIAM J. Optim., vol. 9, no. 1, pp. 33-52, 1998.

[29] L. El Ghaoui, M. Oks, and F. Oustry, "Worst-case value-at-risk and robust portfolio optimization: A conic programming approach," Oper. Res., vol. 51, no. 4, pp. 543-556, Jul. 2003.

[30] W. Hoeffding, "Probability inequalities for sums of bounded random variables," J. Amer. Statist. Assoc., vol. 58, pp. 13-30, 1963.

[31] S. Kanev, B. De Schutter, and M. Verhaegen, "An ellipsoid algorithm for probabilistic robust controller design," Syst. Control Lett., vol. 49, no. 5, pp. 365-375, 2003

[32] L. H. Keel and S. P. Bhattacharyya, "A linear programming approach to controller design," in Proc. IEEE Conf. Decision and Control, San Diego, CA, Dec. 1997, pp. 2139-2148.

[33] P. P. Khargonekar and A. Tikku, "Randomized algorithms for robust control analysis have polynomial time complexity," in Proc. IEEE Conf. Decision on Control, Kobe, Japan, Dec. 1996, pp. 3470-3475.

[34] V. Koltchinskii, C. T. Abdallah, M. Ariola, P. Dorato, and D. Panchenko, "Improved sample complexity estimates for statistical learning control of uncertain systems," IEEE Trans. Autom. Control, vol. 45, no. 12, pp. 2383-2388, Dec. 2000.

[35] C. M. Lagoa, X. Li, and M. Sznaier, "Application of probabilistically constrained linear programs to risk-adjusted controller design," in Proc. Amer. Control Conf., Arlington, VA, Jun. 2001, pp. 738-743.

[36] A. Nemirovski, "Several NP-hard problems arising in robust stability analysis," SIAM J. Matrix Anal. Appl., vol. 6, pp. 99-105, 1993.

[37] Y. Oishi and H. Kimura, "Randomized algorithms to solve parameter-dependent linear matrix inequalities and their computational complexity," in Proc. IEEE Conf. Decision and Control, vol. 2, Dec. 2001, pp. 2025-2030.

[38] A. Prékopa, Stochastic Programming. Norwell, MA: Kluwer, 1995.

[39] L. R. Ray and R. F. Stengel, "A Monte Carlo approach to the analysis of control system robustness," Automatica, vol. 29, pp. 229-236, 1993.

[40] R. T. Rockafellar, Convex Analysis. Princeton, NJ: Princeton Univ. Press, 1970

[41] C. W. Scherer, "Relaxations for robust linear matrix inequality problems with verifications for exactness," SIAM J. Matrix Anal. Appl., vol. 27, no. 2, pp. 365-395, 2005.

[42] R. E. Skelton, T. Iwasaki, and K. Grigoriadis, A Unified Algebraic Approach to Linear Control Design. London, U.K.: Taylor and Francis, 1998.

[43] R. F. Stengel and L. R. Ray, "Stochastic robustness of linear time-invariant control systems," IEEE Trans. Autom. Control, vol. 36, no. 1, pp. 82-87, Jan. 1991

[44] R. Tempo, E. W. Bai, and F. Dabbene, "Probabilistic robustness analysis: Explicit bounds for the minimum number of samples," Syst. Control Lett., vol. 30, pp. 237-242, 1997. 
[45] A. Trofino and C. E. de Souza, "Biquadratic stability of uncertain linear systems," IEEE Trans. Autom. Control, vol. 46, no. 8, pp. 1303-1307, Aug. 2001.

[46] S. Vajda, Probabilistic Programming. New York: Academic, 1972.

[47] V. Vapnik, Statistical Learning Theory. New York: Wiley, 1996.

[48] M. Vidyasagar, A Theory of Learning and Generalization. London, U.K.: Springer-Verlag, 1997.

[49] _ _ "Randomized algorithms for robust controller synthesis using statistical learning theory," Automatica, vol. 37, no. 10, pp. 1515-1528, Oct. 2001.

[50] M. Vidyasagar and V. Blondel, "Probabilistic solutions to some NP-hard matrix problems," Automatica, vol. 37, no. 9, pp. 1397-1405, Sep. 2001.

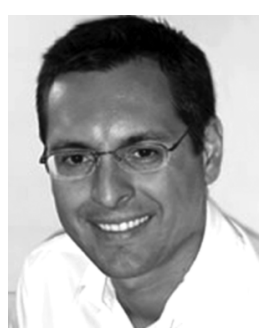

Giuseppe C. Calafiore received the "Laurea" degree in electrical engineering and the Ph.D. degree in information and system theory from Politecnico di Torino, Torino, Italy, in 1993 and 1997, respectively.

He currently serves as an Associate Professor at the Dipartimento di Automatica e Informatica, Politecnico di Torino. He held visiting positions at Information Systems Laboratory, Stanford University, Stanford, CA, in 1995, at Ecole Nationale Superieure de Techniques Avencees (ENSTA), Paris, France, in 1998, and at the University of California, Berkeley, in 1999, where he also had a position as Research Engineer in 2003. His research interests are in the field of analysis and control of uncertain systems, convex optimization, and randomized algorithms. He is author or coauthor of six books and over 70 publications on international journals and conferences.

Dr. Calafiore is Associate Editor for the IEEE TRANSACTIONS ON SYSTEMS, MAN, AND CYBERNETICS.

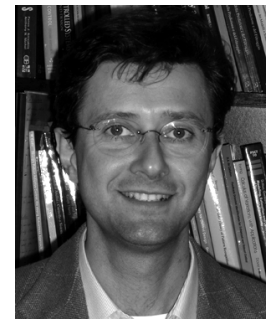

Marco C. Campi was born in Tradate, Italy, on December 7, 1963. He received the doctor degree in electronic engineering from the Politecnico di Milano, Milano, Italy, in 1988.

From 1988 to 1989, he was a Research Assistant with the Department of Electrical Engineering of the Politecnico di Milano. From 1989 to 1992, he worked as a Researcher at the Centro di Teoria dei Sistemi of the National Research Council (CNR) in Milano. Since 1992, he has been with the University of Brescia, Italy, where he is currently a Professor of Automatic Control. He has held visiting and teaching positions at many universities and institutions including the Australian National University, Canberra, Australia; the University of Illinois at Urbana-Champaign; the Centre for Artificial Intelligence and Robotics, Bangalore, India; the University of Melbourne, Australia; and Kyoto University, Japan. His research interests include system identification, stochastic systems, adaptive and data-based control, robust convex optimization, robust control and estimation, and learning theory. His research activity has been conducted through years under many Italian and European projects. Currently, he is leader of the Brescia unit of the European IST project "Distributed control and stochastic analysis of hybrid systems supporting safety critical real-time systems design."

Dr. Campi is an Associate Editor of Systems and Control Letters, and a past Associate Editor of Automatica and the European Journal of Control. He serves as Chair of the Technical Committee IFAC on Stochastic Systems (SS) and is a member of the Technical Committee IFAC on Modeling, Identification and Signal Processing (MISP) and of the Technical Committee IFAC on Cost Oriented Automation. Moreover, he is a Distinguished Lecturer under the IEEE Control Systems Society (CSS) Program. His doctoral thesis was awarded the "Giorgio Quazza" prize as the best original thesis for 1988. 